\title{
Long-term trajectories of SARS-CoV-2 neutralizing antibodies and predictive value of first dose vaccination-induced IgG-antibodies in hemodialysis patients
}

\author{
F. P. Tillmann ${ }^{1,2}$ [ H. Still ${ }^{2}$ Philipp von Landenberg ${ }^{3}$
}

Received: 13 August 2021 / Accepted: 22 November 2021 / Published online: 3 December 2021

(c) The Author(s) 2021

\begin{abstract}
Purpose The predictive value of antibody titers after the first SARS-CoV-2 vaccination and long-term trajectories of antibody titers in hemodialysis patients are unknown.

Methods SARS-CoV-2 IgG antibodies and their neutralizing effect six weeks after the first and second vaccination were analysed in 30 hemodialysis patients. IgG titers served to classify participants as responders or non-responders and to calculate sensitivity, specificity, and accuracy. Associations between potential risk factors and post-vaccine non-response were analysed by Mann-Whitney- $U$ test and Chi-Squared test. Long-term follow-up analysis (ANOVA) on the evolution of neutralizing IgG-titers was performed in 24 participants 94 and 135 days after the second immunization.

Results IgG antibodies $\geq 1 \mathrm{AU} / \mathrm{L}$ (mean $9 \pm 20 \mathrm{AU} / \mathrm{L}$ ) after the first dose were found in 20 patients $(66.7 \%$ ). After the second dose only two participants (6.7\%) remained sero-negative and $16.6 \%$ showed neutralizing levels below $30 \%$, whereas 25 patients showed IgG antibodies with the high neutralizing activity of $86 \pm 18 \%$. Positive IgG antibodies 6 weeks after the first vaccination predicted vaccination effectiveness after two cycles with a specificity of $100 \%$, sensitivity of $76 \%$, and accuracy of $87 \%$. Even low-dose immunosuppressive therapy increased the relative risk for non-response after the first and second dose 1.9 (95\% CI 0.8-4.6) and 4.9 (95\% CI 1.0-23.8) times, respectively. Over a period of about 4.5 months IgG titers slowly declined by $51 \%$ from baseline or by $0.45 \mathrm{AU} / \mathrm{mL}$ per day, respectively.

Conclusion Two cycles of SARS-CoV-2 vaccination-induced high seroconversion rates comparable to the general population. Immunosuppressive medication is a major risk factor for vaccination non-response. Mounted $\operatorname{IgG}$ antibodies showed a high neutralizing capacity as evidence of protective effectiveness. IgG antibodies after the first dose may serve to predict later vaccination outcome. Patients on dialysis display a more rapid decline in antibody titers on long-term follow-up compared to healthy controls.
\end{abstract}

Keywords SARS-CoV-2 $\cdot$ COVID-19 $\cdot$ Vaccination $\cdot$ Neutralizing antibodies $\cdot$ Antibody titer trajectory $\cdot$ Hemodialysis · Long-term follow-up

F. P. Tillmann

frank-peter.tillmann@gmx.de

1 Department of Medicine I-Nephrology, Transplantation \& Medical Intensive Care, Medical Center Cologne-Merheim, University Witten/Herdecke, Ostmerheimer Str. 200, 51109 Cologne, Germany

2 Nephrologisches Zentrum Ibbenbüren, Gravenhorsterstr. 1, 49477 Ibbenbüren, Germany

3 LADR GmbH MVZ Nord-West, Technikerstr. 14, 48465 Schüttorf, Germany

\section{Introduction}

So far different SARS-CoV-2 vaccines have been developed and marketed in different countries around the world. The mode of anti-viral effect of these vaccines can be described as either directly neutralizing or inducing a host immune response via purified virus components, replication-defective viral vector carrying pathogen genes, and mRNA vaccines [1]. In Germany, both gene-based and mRNA-based vaccines have been approved and are in use for several months. Patients on hemodialysis are at high risk of developing severe courses of SARS-CoV-2 infections carrying a high mortality rate $[2,3]$. Despite the high risk in this 
vulnerable patient cohort, hemodialysis patients are vaccinated using the same vaccination scheme as in the general population with two dosages with a specified time interval between the two dosages. Observational reports have shown an insufficient immune response in end-stage-renal disease patients $[4,5]$ as compared to the general population with a reported efficacy of $>90 \%$ after a second dose [6]. Dialysis units are locations with a high risk of acquiring SARS$\mathrm{CoV}-2$ infections making strict hygiene protocols mandatory. Declining viral infection rates have led to calls to reduce the still strict nationwide anti-SARS-CoV-2 measures in many countries. So far, little is known about the efficacy of a first dose anti-SARS-CoV-2 vaccination to mount anti-SARSCoV-2 IgG antibodies and if the standard two-dose fits all vaccination strategy in the general population is sufficiently effective in ESRD patients on hemodialysis. Furthermore, the neutralizing activity and the long-term decline in antibody titers have not yet been fully elucidated in this vulnerable patient cohort. Therefore, this prospective study aimed at describing the effect of SARS-CoV-2 vaccination on the long-term evolution of antibody titers and their neutralizing capacity in a cohort of hemodialysis patients.

\section{Methods}

This is an observational, prospective single-center analysis in hemodialysis in patients $>18$ years of age. Patient classified to participate in this study if they were vaccinated with a first dose of either mRNA SARS-CoV-2 vaccine (BNT162b2, Pfizer-BioNTech) or replication-defective viral vector carrying pathogen gene (ChAdOx $1 \mathrm{nCoV}-19$, OxfordAstraZeneca) at least 3 weeks prior to study inclusion. AntiSARS-CoV-2 antibodies were re-evaluated 6-7 weeks after the second vaccination cycle. Patients with prior SARSCoV-2 infection were not eligible to participate in this study. Post-vaccination analysis included the measurement of SARS-CoV-2 IgG-antibody titers and an evaluation of the neutralizing capacity of the IgG-antibody as described below. Patients had been vaccinated either in central vaccination facilities, by their primary care physicians or the dialysis facility itself. Dates of vaccination, type of vaccination used, and person-related data were stored centrally in a password-protected data sheet.

Past medical history of COVID-19 and outcomes before the start of the study were determined by the medical staff of the facility in all participants prior to the start of the study. Demographic data (age, sex, dialysis vintage, BMI, prior history of transplantation, online conductivity $\mathrm{Kt} / \mathrm{V}$ clearance [OCM-device ${ }^{\mathrm{TM}} \mathrm{Fa}$. Fresenius Medical Care], estimated glomerular filtration rate according to the CKD-EPI formula in $\mathrm{ml} / \mathrm{min} / 1.73 \mathrm{~m}^{2} \mathrm{BSA}$, albumin in $\mathrm{mg} / \mathrm{dl}$, type of hemodialysis access [fistula, graft, catheter], candidacy for renal transplantation, active immunosuppressive medication at the time of antibody titer evaluation, diabetes mellitus, active malignancy, active hepatitis, and anti-HBs-titers, CRP levels in $\mathrm{mg} / \mathrm{dl}$, parathyroid hormone levels in $\mathrm{pmol} / \mathrm{l}$, calcidiol levels in nmol/l, and calcitriol levels in $\mathrm{pg} / \mathrm{ml}$ ) were recorded in every patient at baseline. Major outcome variables were IgG-antibody titers categorized in negative or positive $(\geq 1.0 \mathrm{AU} / \mathrm{ml})$, and the neutralizing capacity of positive antibody titers in $\%(0-<30 \%$ up to $100 \%)$.

The study was approved by the local ethics committee "Ethikkommission der Ärztekammer Westfalen-Lippe und der Westfälischen Wilhelms Universität" in Münster (2021-131-f-S) and conducted in line with the Declaration of Helsinki and the European Union Clinical Trials Directive 2001/20/EC (EU CTD). Written informed consent to participate and to publish was obtained from all individual participants included in the study. All patients gave informed consent prior to study participation.

\section{Laboratory measurements of SARS-CoV-2 antibodies}

\section{SARS-CoV-2 IgG antibody test assay}

In this study, a commercially available immunoassay was used for antibody detection, the anti-SARS-CoV-2 S-RBD IgG (Snibe Diagnostics, New Industries Biomedical Engineering Co., Ltd [Snibe], Shenzhen, China). SARS-CoV-2 $\mathrm{S}-\mathrm{RBD} \operatorname{IgG}$ is a chemiluminescent immunoassay (CLIA) that determines $\mathrm{IgG}$ Ab against the RBD of the Spike (S) protein of the virus, in human serum or plasma. All analyses were performed on MAGLUMI ${ }^{\mathrm{TM}} 4000$ instrument (Snibe Diagnostics), with results expressed in AU/L. The assay has a clinical sensitivity between $74.5 \%$ (days postonset of Symptoms 0-7) and 100.0\% (days post-onset of Symptoms > 15), and a specificity of $99.6 \%$ [95\% confidence interval (95\% CI) $98.7 \%-100.0 \%$ ]. Results were reported in $\mathrm{AU} / \mathrm{L}$ from 0 to 100 . Values greater 100 were reported $>100$ AU/L. For analysis, these data were categorized into three classes of IgG-levels of 0,1-100, and > 100 AU/L. To increase comparability between different investigations data antibody titers in $\mathrm{AU} / \mathrm{mL}$ can be multiplied by 4.3 to receive values in BAU $/ \mathrm{mL}$. Definition of seroconversion or responder status after the second dose:

A value of $\geq 30 \mathrm{AU} / \mathrm{ml}$ about 6 weeks after the second vaccination cycle was considered "seroconversion or responder status" as opposed to "missing or incomplete seroconversion or nonresponder status". 


\section{SARS-CoV-2 IgG neutralizing test assay}

We used the ELISA-based GenScript SARS-CoV-2 Surrogate Virus Neutralization Test Kit (GenScript 105 Biotech, Piscataway Township, USA). The test was used according to the manufacturer's recommendations. Samples were diluted in sample buffer and incubated at $37^{\circ}$ for $30 \mathrm{~min}$ in the 96-well microtiter plates provided, followed by the respective wash and incubation cycles, including controls, and required reagents. The microtiter plates are coated with the "host cell receptor" angiotensin-converting enzyme 2 (ACE2). Samples containing SARS-CoV-2 neutralizing antibodies block the protein-protein reaction between ACE2 and the added (S)-RBD-horseradish peroxidase conjugate. The reduced color change upon the addition of chromogenic substrate can be measured photometrically. Optical density (OD) was measured at $450 \mathrm{~nm}$ using the microplate reader of a VIRCLIA $®$ automation system. The signal to cut-off ratio was calculated and the values printed and interpreted according to the manufacturer's protocol and results were reported in \%. Definition of neutralizing capacity of SARSCoV-2 IgG levels after the second dose: A value of $\geq 30 \%$ about six weeks after the second vaccination cycle was considered "fully neutralizing capacity" as opposed to "missing or incomplete neutralizing capacity".

\section{Statistical analyses}

Data are shown as mean plus-minus standard deviation (SD) or percentage, according to the type of variable analysed. We used the Chi-Squared test for associations between qualitative variables and the Mann-Whitney- $U$ test for quantitative variables. One-way ANOVA with post-hoc Bonferroni adjustment for multiple comparisons was applied to test for differences in SARS-CoV-2 IgG-titers over long-term follow-up. Values of $p<0.05$ were considered statistically significant. Statistical analyses were performed using SPSS, IBM Corp., Armonk, NY.

\section{Results}

\section{Patient characteristics}

In total 30 patients, 22 men (73.3\%) and 8 women (26.7\%), were included in this analysis. Three patients $(10.0 \%)$ were anti-HBcore-antibody positive and only one patient suffered from active malignancy of the prostate. Four patients had a failing renal transplant in situ. Seven patients were dialyzed via a central dialysis catheter. Seven patients (23.3\%) received active but low-dose immunomodulatory therapy in form of steroids alone $(n=2)$, a combination of steroids and calcineurin-inhibitors $(n=4)$ or a combination of steroids and cyclophosphamide $(n=1)$. No patient received mycophenolate or its derivatives. SARS-CoV-2 vaccination was performed in $28(93.3 \%)$ patients via mRNA-based and only two via vector-based vaccines. All except two patients were dialyzed via polysulfone membranes. Further cohort characteristics are shown in Table 1.

\section{SARS-CoV-2 IgG antibody titers and neutralizing capacity after the first dose}

About 6-7 weeks after application of the first dose the mean SARS-CoV-2 IgG antibody titer was $9 \pm 20 \mathrm{AU} / \mathrm{L}$, whereas $10(33.3 \%)$ patients had no detectable antibody titers. Only six patients $(20 \%)$ showed neutralizing levels over $30 \%$ (mean $50 \pm 20 \%$ ), whereas 24 patients showed no measurable neutralizing activity. Despite the relatively low titer 20 patients $(66.7 \%)$ were already able to mount antibodies despite only on the cycle of vaccination. Without exception patients with a positive IgG titer after the first dose were
Table 1 Basic cohort characteristics

\begin{tabular}{llll}
\hline Cohort of 30 hemodialysis patients & & & \\
\hline Male sex (\%) & 73.3 & Kt/V & $1.2 \pm 0.4$ \\
\hline Vaccine type (\%) & mRNA 90 & eGFR & $8 \pm 5$ \\
Pector 10 & & $3566 \pm 618$ \\
Prior KTx (\%) & 13.3 & Albumin g/L & $62.3 \pm 15.3$ \\
HD-catheter (\%) & 23.3 & Age in years & $44 \pm 17$ \\
KTx waitlisted (\%) & 46.7 & Days 1te to 2te vaccination & $3.82 \pm 3.55$ \\
IS-therapy (\%) & 23.3 & HD-vintage in years & $0.6 \pm 0.8$ \\
Diabetes (\%) & 26.7 & CRP mg/dL & $36 \pm 37$ \\
Active malignancy & 3.3 & PTH pmol/L & $72 \pm 21$ \\
Positive anti-HBs-titer (\%) & 46.7 & Calcidiol nmol/L & $17 \pm 7$ \\
BMI & $26.0 \pm 5.3$ & Calcitriol pg/mL & \\
\hline
\end{tabular}

$\mathrm{HD}=$ hemodialysis, $\mathrm{KTx}=$ kidney transplantation, $\mathrm{IS}=$ immunosuppressive, eGFR $=\mathrm{CKD}$-EPI formula in $\mathrm{ml} / \mathrm{min} / 1.73 \mathrm{~m}^{2} \mathrm{BSA}$ 
all able to mount a full antibody response after the second immunization cycle.

\section{SARS-CoV-2 IgG antibody titers and neutralizing capacity after the second dose}

About 6-7 weeks after application of the second dose 21 (70.0\%) patients had antibody titers $>100$ AU/L, seven patients $(23.3 \%)$ showed titers between 1 and $100 \mathrm{AU} / \mathrm{L}$, and only two patients $(6.7 \%)$ had no detectable antibodies. Only five patients (16.6\%) showed neutralizing levels below $30 \%$, whereas 25 patients showed IgG antibodies with the high neutralizing activity of $86 \pm 18 \%$. The 20 patients who were able to mount an antibody response already after the first vaccination cycle showed an even higher neutralizing effect of $91 \pm 17 \%$ after the second dose.

\section{Characteristics of incomplete and/ or non-responders after the first dose}

Contrary to the definition of missing or incomplete response after the second dose, non-response after the first dose was defined as 0 or non-detectable $\operatorname{IgG}$ antibodies prior to application of the second vaccination. 20 patients showed positive IgG antibody titers, whereas in ten patients no sero-reactivity was detectable after the first dose. Analysis of continuous (Mann-Whitney- $U$ test) variables did not show any difference between both regarding the following variables: $\mathrm{BMI}$, age, Kt/V, eGFR, dialysis vintage, albumin, c-reactive protein, parathyroid hormone, calcidiol, and calcitriol levels. Patients on immunomodulatory therapy showed a 1.9 (95\% CI 0.8-4.6) times higher relative risk of "non-response status" to vaccination as compared to patients without. Analysis of categorical variables was not possible due to cell-numbers $\geq 5$.

\section{Characteristics of incomplete and/ or non-responders after the second dose}

In sum, five patients (16.6\%, three men and two women) were classified as "missing or incomplete seroconversion or non-responder status". The same patients were also classified as "missing or incomplete neutralizing capacity status". Nevertheless, only two patients showed no detectable antibody response after full vaccination at all. Of note, three of these patients were on an immunosuppressive regimen consisting of steroids and calcineurin-inhibitors. Non-parametric testing (Mann-Whitney $U$ test) only pointed towards a possible difference in dialysis vintage between responders and non-responders $(4.31 \pm 3.66$ vs. $1.37 \pm 1.39$ years, $p=0.037)$, whereas no signal was found for BMI, age, Kt/V, eGFR, albumin, c-reactive protein, parathyroid hormone, calcidiol, and calcitriol levels. Patients on immunomodulatory therapy showed a 4.9 (95\% CI 1.0-23.8) times higher relative risk of "incomplete or non-response status" to vaccination as compared to patients without. Analysis of categorical variables was not possible due to cell-numbers $\geq 5$.

\section{Predictive value of IgG-antibody positivity on vaccination effectiveness after two dosages}

In a further step, we tested the performance characteristics of a determination of SARS-CoV-2 IgG antibody measurement 6-7 weeks after the first vaccination cycle regarding vaccine efficacy 6-7 weeks after completion of the full vaccination schedule. Under the assumption of a positive test result in cases of IgG antibody $>1 \mathrm{AU} / \mathrm{L}$ and positive outcome result in cases of IgG levels $>30 \mathrm{AU} / \mathrm{L}$ we obtained the following results: sensitivity $76 \%$, specificity $100 \%$, false-positive-rate $0 \%$, false-negative-rate $55 \%$, and accuracy $87 \%$.

\section{Long-term evolution of IgG-antibodies and their effectiveness after two dosages}

From the initial 30 participants of the cohort 24 patients were further analysed with respect to long-term evolution of IgG-antibody titers and their neutralizing capacity after two standard dose vaccinations. Six patients could not be evaluated due to the following reasons: one patient died due to acute pancreatitis, two patients received a booster vaccination in the meantime, and three patients were classified as non-responders. The evolution of IgG-antibodies in AU/ $\mathrm{mL}$ and their neutralizing capacities are shown in Table 2. One way ANOVA with post-hoc Bonferroni adjustment of IgG-antibody titers showed significant differences in antibody titers $(p \leq 0.001)$ with the exceptions of comparisons

Table 2 Long-term evolution of SARS-CoV-2 IgG antibodies in AU/ $\mathrm{mL}$ and the respective neutralizing effect in 24 patients on hemodialysis

\begin{tabular}{llllll}
\hline \multicolumn{6}{l}{ Long-term follow-up data in 24 hemodialysis patients } \\
\hline \multirow{4}{*}{ IgG } & 1 & 2 & 3 & 4 \\
& Mean & 11 & 91 & 67 & 46 \\
& STD & 22 & 24 & 29 & 36 \\
\multirow{4}{*}{ cPASS } & $95 \%$ CI & $2-21$ & $80-101$ & $51-84$ & $31-61$ \\
& Mean & 25 & 83 & 69 & 65 \\
& STD & 20 & 26 & 30 & 25 \\
\multirow{4}{*}{ Days } & $95 \%$ CI & $17-34$ & $72-94$ & $57-82$ & $54-76$ \\
& Mean & 44 & 35 & 94 & 135 \\
& STD & 19 & 19 & 32 & 36 \\
& $95 \%$ CI & $35-52$ & $27-43$ & $80-108$ & $120-150$ \\
\hline
\end{tabular}

Measurement 1 was performed on average 11 days after the first vaccination, measurements $2-4$ were performed on average 35,94 , and 135 days after the second standard dose vaccination 


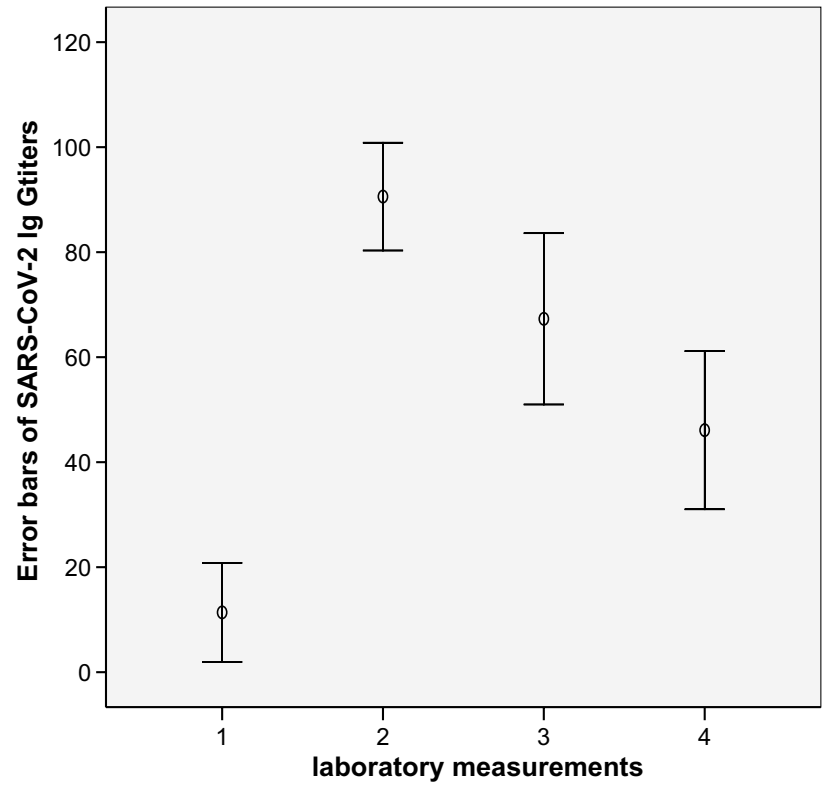

Fig. 1 Error bars of SARS-CoV-2 IgG antibodies showing the means and respective $95 \%$ CI over 4 measurement cycles on long-term follow-up in 24 hemodialysis patients, one way ANOVA $p<=0.001$ with the exceptions of comparisons between the second and third measurements $(p=0.065)$ and between the third and fourth measurement $(p=0.120)$
Table 3 Decline in antibody titers between the second, third and fourth laboratory measurements in 24 hemodialysis patients in $\%$ and $\mathrm{AU} / \mathrm{mL}$ per day

\begin{tabular}{lll}
\hline $\begin{array}{l}\text { Decline in SARS-CoV IgG } \\
\text { antibody titers }\end{array}$ \\
\hline $\begin{array}{l}\text { \% from } \\
\text { baseline }\end{array}$ & AU/mL/day \\
\hline 2 to 3 & 74 & -0.41 \\
3 to 4 & 69 & -0.56 \\
2 to 4 & 51 & -0.45 \\
\hline
\end{tabular}

Values six weeks after the second vaccination were taken as baseline value

between the second and third laboratory measurements, and between the third and fourth evaluation (respective error bars are shown in Fig. 1). Declining rates of antibody titers between the laboratory measurements are shown in Table 3 .

\section{Discussion}

This study aimed to investigate the effectiveness of the first SARS-CoV-2 vaccination cycle on the evolution of antivirus antibodies and their neutralizing capacity in hemodialysis patients. Our results are in line with a very high effectiveness of mRNA- and vector-based vaccines as reported in the general population. In our cohort of hemodialysis patients only $6.7 \%$ were classified as non-responders without any antibody formation and only $9.9 \%$ showed a reduced evolution of neutralizing antibodies between 1 and $30 \mathrm{AU} / \mathrm{mL}$. Our response rates after the second vaccine dose are in line with previous results in ESRD patients on hemodialysis e.g., Anand and co-workers reported a reduced effectiveness in $26.5 \%$ of participants 28 days after the second dose in 355 hemodialysis patients [7]. Other investigations found response rates of over $80 \%$ [8] or over $90 \%$ [9-11]. These data are comparable or even superior to antibody response rates in symptomatic hemodialysis patients after infection with the wild-type virus [12]. Interestingly, serial antibody measurements in 122 hemodialysis patients after infection showed raising titers with a peak at four months [13]. Therefore, the timing of antibody titer measurement might be a relevant potential source of discrepancies between investigations partly explaining differences in rates of seroconversion also after vaccination. Our study adds to these reports so far, as we could not only confirm the presence of SARSCoV-2 antibodies in $93.3 \%$ of our participants but also their neutralizing capacity with a mean value of $86 \%$ after two vaccination cycles.

In contrast to the growing number of studies on vaccine efficacy after a full vaccination with two dosages, the data on the effect after a first vaccination in hemodialysis patients is still scarce. The first report on first-dose effects in hemodialysis patients was reported by Torregiani and co-workers in April this year [14]. They reported a mean antibody titer of $8 \mathrm{U} / \mathrm{ml}$ comparable to our findings of $9 \mathrm{AU} / \mathrm{mL}$ but found only a reduced responder rate of $35.6 \%$ as opposed to our rate of $66.6 \%$. A reason for this difference might be the reduced time-interval of three weeks between application of the first dose and antibody measurement in their study and 6-7 weeks in our investigation. Other investigations reported sero-conversion rates of $79.8 \%$ four weeks after the first dose of BNT162b2 mRNA vaccine in 94 hemodialysis patients [15] or $42 \%$ in 50 patients in a study by Zitt and co-workers [16]. Of note, the authors of the latter applied a different definition of sero-conversion using the assay-specific cutoff value of 33.8 binding activity units per milliliter four weeks after the first dose [16]. Therefore, in interpreting and comparing study results not only the timing of antibody determination with respect to vaccination but also different definitions of response and non-response should be considered. Our definition of a positive response of an antibody titer $>0 \mathrm{AU} / \mathrm{mL}$ after the first dose of vaccination showed promising performance parameters with a specificity of $100 \%$ and an accuracy of $87 \%$. Immunosuppressive therapy even at low doses increase to risk for non-response to vaccination in our cohort to 1.9 and 4.9 times after the first and second vaccination dose, respectively. Therefore, in our view, the purpose of checking the effect after only one dose could be to offer non-responding vaccine recipients a modified vaccination schedule or a reduction in any 
immunosuppressive therapy already at this early stage. The slight statistical association between fewer years on hemodialysis and lower vaccine response is, in our opinion, more likely since patients with recent graft failure some months ago were still on low-dose immunosuppression. Finally, we could not detect an association between antibody response and vitamin D levels or success to hepatitis B vaccination.

Major limitations of our investigation are the reduced patient numbers and the single-center observational non-randomized design of our study protocol. Furthermore, severe selection bias towards patients interested in participating in this investigation might have occurred.

As opposed to one report of hemodialysis patients after wild virus infection [13], we could demonstrate a continuous fall in antibody titers over a period of 4.5 months after the second vaccination dose. Recently, long-term data on titer progression have also been published. For example, Levin and co-workers were able to describe the IgG titer trajectories of neutralizing SARS-CoV-2 antibodies over six months in a large cohort of employees of a health facility [17]. Interestingly, their data are comparable to the results of this investigation in a greater and more rapid decline in total IgG-titers as compared to neutralizing antibodies. Another investigation reported an estimated anti-spike IgG half-life of 184 days in a representative population-based cohort of over 7.000 participants in the United Kingdom [18]. Another analysis reported an even more pronounced but expected fall in IgG titers comparable to titers after the first vaccination dose in 122 healthy volunteers after six months [19]. Generally, this fall is linked to the fact, that vaccination does normally only induce short-lived plasma cells as compared to log-lived plasma cells after wild virus infections. Most recently, Speer and co-workers analysed antibody trajectories in 124 hemodialysis and 41 peritoneal dialysis patients [20]. This prospective multi-center study found a decline in IgG antibody titers after 12 weeks of 55\% in participants on hemodialysis and $45 \%$ in patients on peritoneal dialysis. Therefore, these data combined with our findings of an IgG antibody titer half-life of about 135 days are suggestive of a more rapid decline in antibody titers in hemodialysis patients. Despite reports on mild clinical courses of wild-type infections after vaccination [21], no conclusive information on the effect of falling titers on break-through infections in hemodialysis patients have yet been published. Consequently, repeat antibody testing and booster vaccinations seem to be a sensible strategy in ESRD patients given the high vulnerability of this patient population to dismal clinical courses or even death after SARS-CoV-2 infection.

In conclusion, our results confirmed high antibody titer conversion rates after two full cycles of SARS-CoV-2 vaccination and showed the neutralizing capacity of the mounted antibodies. Even low-dose immunosuppressants seem to impair a protective antibody response in hemodialysis patients. Hemodialysis patients seem to be prone to a more rapid decline in antibody titers as compared to healthy cohorts. Continuous antibody surveillance and probably booster vaccinations might serve in the near future to protect these patients from unfavorable clinical courses after SARS. coV-2 contact or break-through infections.

Acknowledgements Were are grateful to the staff and personnel of "Nephrologisches Zentrum Ibbenbüren" and "LADR-Laborzentrum Schüttorf" in helping to collect the data and perform the vaccinations.

Author contributions FPT designed the study, collected the data, analysed the data, and drafted the manuscript. HS performed the data acquisition and conceived the study. PvL designed the study, performed the laboratory analyses, conceived the study, and helped in drafting the manuscript. All authors have read and approved the final manuscript.

Funding Open Access funding enabled and organized by Projekt DEAL.

\section{Declarations}

Conflict of interest All of the authors declare that they have no conflict of interest.

Ethical approval All of the procedures performed in studies involving human participants were in accordance with the ethical standards of the institutional and/or national research committee and with the 1964 Helsinki Declaration and its later amendments or comparable ethical standards.

Open Access This article is licensed under a Creative Commons Attribution 4.0 International License, which permits use, sharing, adaptation, distribution and reproduction in any medium or format, as long as you give appropriate credit to the original author(s) and the source, provide a link to the Creative Commons licence, and indicate if changes were made. The images or other third party material in this article are included in the article's Creative Commons licence, unless indicated otherwise in a credit line to the material. If material is not included in the article's Creative Commons licence and your intended use is not permitted by statutory regulation or exceeds the permitted use, you will need to obtain permission directly from the copyright holder. To view a copy of this licence, visit http://creativecommons.org/licenses/by/4.0/.

\section{References}

1. Windpessl M, Bruchfeld A, Anders HA et al (2021) COVID-19 vaccines and kidney disease. Nat Rev Nephrol 17:291-293

2. Savino M, Casula A, Santhakumaran S, et al. Sociodemographic features, and mortality of individuals on haemodialysis treatment who test positive for SARS-CoV-2: a UK Renal Registry data analysis. PLoS One. 2020;15:e02411263.

3. Hoxha E, Suling A, Turner JE et al (2021) COVID-19 prevalence and mortality in chronic dialysis patients. Dtsch Arztebl Int 118:195-196

4. Schrezenmeier E, Bergfeld L, Hillus D et al. Immunogenicity of COVID-19 tozinameran vaccination in patients on chronic dialysis. Front Immunol 2021;12:690698.

5. Grupper A, Sharon N, Finn T et al (2021) Humoral response to the Pfizer BNT162b2 vaccine in patients undergoing maintenance 
hemodialysis. Clin J Am Soc Nephrol. https://doi.org/10.2215/ CJN.03500321

6. Dagan N, Barda N, Kepten E, Miron O, Perchik S, Katz MA, Hernán MA, Lipsitch M, Reis B, Balicer RD (2021) BNT162b2 mRNA Covid-19 vaccine in a nationwide mass vaccination setting. N Engl J Med 384:1412-1423

7. Anand S, Montez-Rath M, Han J, Garcia P, Cadden L, Hunsader P, Kerschmann R, Beyer P, Dittrich M, Block G, Boyd S, Parsonnet J, Chertow G (2021) Antibody Response to COVID-19 Vaccination in Patients Receiving Dialysis. J Am Soc Nephrol. https://doi. org/10.1681/ASN.2021050611

8. Attias P, Sakhi H, Rieu P, Soorkia A, Assayag D, Bouhroum S, Nizard P, El Karoui K (2021) Antibody response to the BNT162b2 vaccine in maintenance hemodialysis patients. Kidney Int 99:1490-1492

9. Berar Yanay N, Freiman S, Shapira M, Wishahi S, Hamze M, Elhaj M, Zaher M, Armaly Z (2021) Experience with SARSCoV-2 BNT162b2 mRNA vaccine in dialysis patients. Kidney Int 99:1496-1498

10. Broseta JJ, Rodriguez-Espinosa D, Rodriguez N, Del Mar MM, Marcos AM et al (2021) Humoral and Cellular Responses to mRNA-1273 and BNT162b2 SARS-CoV-2 Vaccines Administered to Hemodialysis Patients. Am J Kidney Dis 0272 6386(21):00689-00692. https://doi.org/10.1053/j.ajkd.2021.06. 002

11. Frantzen L, Cavaille G, Thibeaut S, El-Haik Y. Efficacy of the BNT162b2 mRNA Covid-19 Vaccine in a hemodialysis cohort. Nephrol Dial Transplant 2021;gfab165. doi: https://doi.org/10. 1093/ndt/gfab165

12. Alcázar-Arroyo R, Portolés J, López-Sánchez P et al (2021) Rapid decline of anti-SARS-CoV-2 antibodies in patients on haemodialysis: the COVID-FRIAT study. Clin Kidney J 14:1835-1844

13. Forbes S, Davari M, Gnanasampanthan S et al. Persistence of antibody response to SARS-CoV-2 in a cohort of haemodialysis patients with COVID-19. Nephrol Dial Transplant 2021; gfab066. doi: https://doi.org/10.1093/ndt/gfab066

14. Torreggiani M, Blanchi S, Fois A et al (2021) Neutralizing SARS$\mathrm{CoV}-2$ antibody response in dialysis patients after the first dose of the BNT162b2 mRNA COVID-19 vaccine: the war is far from being won. Kidney Int 99:1494-1496

15. Billany RE, Selvaskandan H, Adenwalla SF et al (2021) Seroprevalence of antibody to $\mathrm{S} 1$ spike protein following vaccination against COVID-19 in patients receiving hemodialysis: a call to arms. Kidney Int 99:1492-1494

16. Zitt E, Davidovic T, Schimpf J et al. The Safety and Immunogenicity of the mRNA-BNT162b2 SARS-CoV-2 Vaccine in Hemodialysis Patients. Front Immunol 2021;12:704773.

17. Levin EG, Lustig $\mathrm{Y}$, Cohen $\mathrm{C}$ et al. Waning Immune Humoral Response to BNT162b2 Covid-19 Vaccine over 6 Months. N Engl J Med 2021;NEJMoa2114583.doi: https://doi.org/10.1056/ NEJMoa2114583.

18. Wei J, Matthews PC, Stoesser N et al (2021) Anti-spike antibody response to natural SARS-CoV-2 infection in the general population. Nat Commun 12:6250. https://doi.org/10.1038/ s41467-021-26479-2

19. Naaber P, Tserel L, Kangro $K$ et al. Dynamics of antibody response to BNT162b2 vaccine after six months: a longitudinal prospective study. Lancet Reg Health Eur. 2021;100208. doi:https://doi.org/10.1016/j.lanepe.2021.100208.

20. Speer C, Schaier M, Husshag C et al (2021) Longitudinal humoral responses after COVID-19 vaccination in peritoneal and hemodialysis patients over twelve weeks. Vaccines (Basel) 9:1130. https://doi.org/10.3390/vaccines9101130

21. Tillmann FP, Plassman G, Ricken J et al (2021) Clinical course of COVID-19 infection in a hemodialysis patient despite prior SARS-CoV-2 vaccination: A case report. Arch Nephrol 4:7-10. https://doi.org/10.22259/2639-3573.0401002

Publisher's Note Springer Nature remains neutral with regard to jurisdictional claims in published maps and institutional affiliations. 